\title{
Pentalogía de Cantrell. Presentación de un caso clínico
}

\author{
ANDREA PARRA B. ${ }^{1}$, ELIANA GÓMEZ C. ${ }^{1}$, \\ FELIPE A. PALACIO D. ${ }^{2}$, MARÍA TERESA GARCÍA V. ${ }^{3}$ \\ 1. Residentes de Pediatría, Universidad Pontificia Bolivariana, Colombia. \\ 2. Pediatra, Unidad de Cuidados Intensivos Neonatales, Clínica Universitaria Bolivariana, Colombia. \\ 3. Neonatóloga, Unidad de Cuidados Intensivos Neonatales, Clínica Universitaria Bolivariana, Colombia.
}

\begin{abstract}
Pentalogy of Cantrell. Clinical case

Pentalogy of Cantrell is a rare disease that results from alterations in embryo development. It is characterized by defects of the lower sternum, midline supraumbilical abdominal wall defect, deficiency of the anterior diaphragm, defects in the diaphragmatic pericardium and cardiac anomaly. Case Report: The case of a male neonate with a prenatal diagnosis of abdominal wall defect is presented, who was subsequently diagnosed with Pentalogy of Cantrell. This study emphasizes the prenatal diagnosis possibilities and the poor prognosis for these patients when their clinical presentation is associated with severe lung hypoplasia.

(Key words: Congenital heart disease, abdominal wall defect, Pentalogy of Cantrell).

Rev Chil Pediatr 2013; 84 (5): 532-536
\end{abstract}

\section{RESUMEN}

La pentalogía de Cantrell es una enfermedad poco frecuente que resulta de alteraciones embriológicas del mesodermo, caracterizada por defecto en la porción baja esternal, defecto de la pared abdominal supraumbilical, defecto diafragmático anterior, defecto del pericardio diafragmático y anomalía cardiaca. Caso clínico: Se presenta el caso de un neonato de sexo masculino con diagnóstico prenatal de defecto de la pared abdominal, en quien posteriormente se realizó el diagnóstico de Pentalogía de Cantrell. Al presentar este caso se destaca la posibilidad diagnóstica en la vida fetal, y el pobre pronóstico de estos pacientes cuando su presentación clínica se asocia a hipoplasia pulmonar severa.

(Palabras clave: Cardiopatía congénita, defecto de la pared abdominal, pentalogía de cantrell).

Rev Chil Pediatr 2013; 84 (5): 532-536

Recibido el 3 de febrero de 2013, devuelto para corregir el 23 de marzo de 2013, segunda versión 18 de junio de 2013, aceptado para publicación el 6 de septiembre de 2013.

Este trabajo cumple con los requisitos sobre consentimiento /asentimiento informado, comité de ética, financiamiento, estudios animales y sobre la ausencia de conflictos de intereses según corresponda.

Correspondencia a:

Andrea Parra Buitrago

E-mail: andreaparrab@gmail.com 


\section{Introducción}

La pentalogía de Cantrell es una rara combinación de defectos de la línea media supra umbilical, descrito por Rivera et al. en el año 1958. Es una enfermedad poco frecuente la cual consiste en ectopia cordis toracoabdominal e involucra 5 defectos congénitos asociados 1) hendidura de la parte inferior del esternón; 2) defecto de la pared abdominal; 3) deficiencia anterior del diafragma; 4) defectos intracardiacos; 5) defectos pericardio diafragmáticos. Son más afectados los varones en una proporción de 2:1 y suelen tener sintomatología más severa que en las mujeres.

Los defectos de pared abdominal ocurren en 1 de cada 2.000 nacidos vivos, y los defectos abdominales más comunes de la pared son la gastrosquisis y el ónfalocele. La presencia de un ónfalocele se ha asociado con enfermedades como la pentalogía de Cantrell, el síndrome de banda amniótica, y el síndrome de Beckwith-Wiedemann.

Estos defectos congénitos pueden ser diagnosticados tan pronto como en el primer trimestre del embarazo. El diagnóstico prenatal de los defectos congénitos y la posibilidad de monitoreo fetal permite planificar con precisión el momento de la atención inicial del recién nacido y planear la mejor opción de tratamiento. La ecografía y la resonancia magnética nuclear posnatales suelen ser de utilidad una vez que se sospecha su presencia.

Se presenta a continuación un caso clínico que debido a la combinación de defecto en la pared abdominal, defecto del diafragma anterior, pericardio diafragmático, defecto cardíaco, y alteración en la longitud del esternón se le diagnóstico la pentalogía de Cantrell, en el cual el pronóstico fue desfavorable, ya que además de las anomalías existentes estaba asociado el riesgo de la hipoplasia pulmonar.

El objetivo de esta comunicación es informar de una patología de baja prevalencia, en la cual es posible hacer el diagnóstico durante la vida fetal. Se expone el curso clínico y su manejo demostrando la BAJA tasa de supervivencia cuando existen malformaciones cardíacas importantes o asociación con hipoplasia pulmonar.

\section{Caso clínico}

Neonato nacido a término de $38+2$ semanas de edad gestacional, peso de $3.170 \mathrm{~g}$, talla $50 \mathrm{~cm}$, apgar 7/10, con antecedente de defecto en pared abdominal gigante, $\mathrm{y}$ falta de integridad diafragmática, por ecografía prenatal.

Nació con defecto en pared abdominal gigante de $5 \mathrm{~cm} \times 5 \mathrm{~cm}$ aproximadamente, que se cubrió con apósito estéril tipo bolsa de polivinilcloruro, y se hospitalizó en Unidad de Cuidados Intensivo Neonatal (UCIN) para valoración por cirugía y atención multidisciplinaria.

Durante el segundo día de vida en ocasiones presentó desaturaciones hasta $82 \%$, con mejoría rápida, pero paulatinamente se acompañó de taquipnea y retracciones, con dificultad respiratoria progresiva y cianosis, por lo cual se decidió soporte ventilatorio con ventilación mecánica invasiva. Con diagnóstico asociado de hipoplasia pulmonar, requirió progresivamente aumento de parámetros y se cambió a modo ventilatorio de alta frecuencia como estrategia de protección, posteriormente fue candidato para óxido nítrico según protocolo institucional.

Se le realizó estudio ecográfico completo para descartar malformaciones asociadas, ecografía cerebral y renal normales, y ecocardiografía que muestra dextro posición, presencia de foramen oval permeable (FOP), insuficiencia valvular mitral, insuficiencia de la válvula tricúspide leve e hipertensión pulmonar moderada a severa, presencia de imagen compatible con hígado a nivel del tórax.

Valorado por cirugía con diagnóstico de ónfalocele y comunicación pericardio-peritoneal, se programó para cirugía. A través del ónfalocele, el lóbulo hepático izquierdo e intestinos eran visibles. (figura 1). El esternón era anatómicamente completo, pero más corto.

El primer tiempo quirúrgico se realizó al $2^{\circ}$ día de vida para reinserción y fijación del silo (figura 2).

Al $7^{\circ}$ día de vida, luego del segundo tiempo quirúrgico en el cual se inició el cierre progresivo de la cavidad abdominal y reparación de la ventana pericardio diafragmática definitiva, el paciente mejoró la saturación con el inicio de la ventilación de alta frecuencia y el óxido 


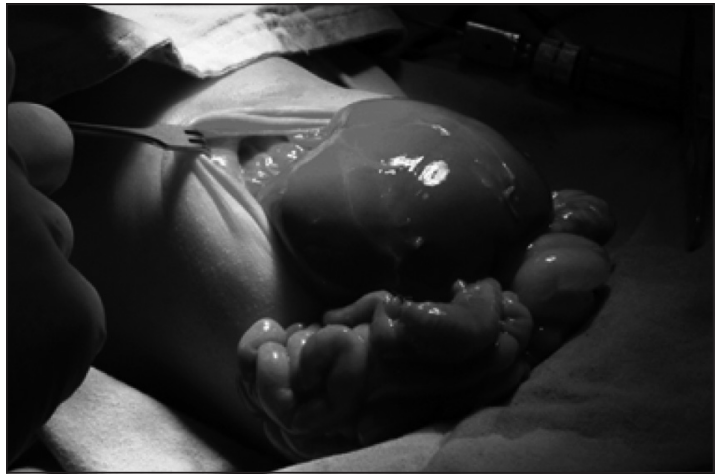

Figura 1. Onfalocele con exposición del lóbulo hepático izquierdo e intestino.

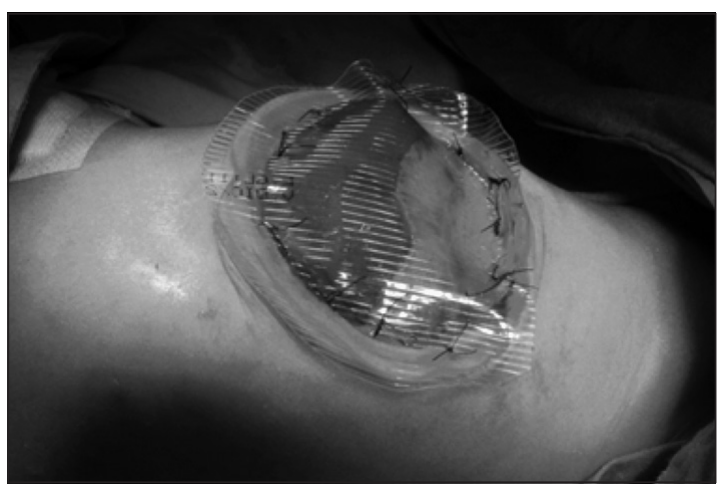

Figura 2. Intervención quirúrgica del paciente. Recién nacido con onfalocele, se observa el silo colocado en el defecto de la pared abdominal.

nítrico, pero aumentó el nivel de parámetros de fase aguda, por lo que requirió cambio de antibiótico y cultivos. Dado una hemoglobina en el límite inferior (tabla 1), se realizó transfusión de glóbulos rojos.

A los 14 días de vida, con ónfalocele cerrado parcialmente con parche en la piel y con defecto pericardio diafragmático anterior cerrado con parche, continuaba críticamente enfermo con soporte inotrópico y ventilatorio, pero permitiendo retiro de óxido nítrico según protocolo. Y se le realiza una última intervención quirúrgica a los 16 días de vida para cierre definitivo y remodelación de la pared abdominal.

A pesar de que no se encontró aislamiento microbiano en los cultivos y no hubo descompensación desde el punto de vista infeccioso, el paciente persistió con difícil manejo ventilatorio, sumado a las malformaciones descritas
Tabla 1. Evolución analítica

\begin{tabular}{|ccccc|}
\hline Hb & Hto & Leucocitos & Plaquetas & PCR \\
\hline 18,9 & 56 & 18.650 & 271.000 & 1 \\
\hline 15,7 & 47 & 11.340 & 235.000 & 1,8 \\
\hline 13,7 & 41,2 & 7.560 & 94.100 & 2,8 \\
\hline 11,3 & 32,9 & 10.780 & 220.900 & 15,6 \\
\hline 10 & 33 & 12.080 & 407.000 & 4,9 \\
\hline
\end{tabular}

Hb: Hemoglobina; Hto: hematocrito; PCR: proteína C reactiva.

previamente, la hipertensión pulmonar, por lo cual a pesar de los procedimientos quirúrgicos fallece al veinte y un día de vida.

\section{Discusión}

En 1958, Cantrell y cols, describieron un síndrome poco común en el cual se integran cinco anomalías: un defecto de la pared abdominal en la línea media, un defecto esternal inferior, un defecto en el diafragma anterior, un defecto pericárdico diafragmático y anormalidades congénitas del corazón ${ }^{1}$. Aunque la ectopia cordis toracoabdominal (comunicación directa del corazón entre el tórax y la cavidad abdominal) está frecuentemente asociada con pentalogía de Cantrell, puede o no estar presente ${ }^{2}$.

Desde entonces hasta la fecha se han publicado un total de 160 casos en la literatura ${ }^{3}$.

La frecuencia de la enfermedad es baja: 2 de 1.716 autopsias con una prevalencia reportada de 0,079 por cada 10.000 nacidos vivos ${ }^{4,5}$.

Es de importancia la presentación de este caso clínico debido a esta poca prevalencia, demostrándose la posibilidad diagnóstica desde la vida fetal y la poca supervivencia a pesar del manejo multidisciplinario, más en los pacientes con cardiopatía asociada e hipertensión pulmonar severa como se muestra en este paciente.

Se tiene certeza de que es un síndrome producto de trastornos del mesodermo y se han encontrado formas completas, incompletas y fenotipos atípicos y complejos de la pentalogía, así como la presencia de numerosas anomalías asociadas: desde defectos de extremi- 
dades hasta infarto de bazo y riñones, atresia intestinal, perforación intestinal, síndrome de intestino corto, ano imperforado, agenesia de vesícula biliar y tetralogía de Fallot ${ }^{6,7}$. A su vez anormalidades cromosómicas, paladar hendido e hipoplasia pulmonar ${ }^{8,9}$.

Esta entidad presenta asociación con cardiopatías congénitas en el $80 \%$. Las más comunes han sido: CIV (100\%), CIA (53\%), EP (33\%), tetralogía de Fallot $(20 \%)^{10,11}$.

Sin embargo, también se ha escrito su asociación con la atresia pulmonar, atresia mitral, transposición de los grandes vasos, doble vía de salida del ventrículo derecho, drenaje venoso anómalo y ventrículo único con y sin estenosis pulmonar ${ }^{12}$.

El manejo obstétrico debe incluir una búsqueda exhaustiva de las anomalías asociadas sobre todo las cardíacas mediante ecocardiografía fetal y la realización de cariotipo ${ }^{13}$.

Respecto al manejo de la pentalogía, es importante mencionar que debido al poco tejido en las estructuras que debieron cerrarse y que no lo concretaron, la reparación quirúrgica consiste en acabar lo que el crecimiento y desarrollo normales no hicieron ${ }^{14}$.

La mayor parte de los niños que fallecen dentro de las primeras horas de vida, ocurre como consecuencia del gran defecto de la pared anterior del tórax y abdomen como ruptura de vísceras abdominales expuestas durante el parto, dificultades respiratorias por herniación de vísceras, sepsis e insuficiencia cardíaca ${ }^{15}$. De ahí que el manejo oportuno de los órganos expuestos mediante solución estéril y de antibióticos de amplio espectro cuando se trate de la ectopia cordis torácica así como de cubrir con material protésico hipoalergénico, modificará sustancialmente el pronóstico de la enfermedad $^{16}$.

En general el pronóstico es desfavorable, ya que además de las anomalías existentes gravita el riesgo de hipoplasia pulmonar bilateral, en ocasiones incompatible con la vida ${ }^{17}$. La tasa de sobrevivencia no sobrepasa el 5\% ${ }^{18-21}$.

\section{Conclusión}

La etiología del síndrome se desconoce, pero la anormalidad primaria se produce antes de los 21 días de vida fetal, con insuficiencia en la fusión de la línea media del septo transverso del diafragma. Lo que conduce a la falta de cierre de la pared abdominal. Por lo que se hace de vital importancia la realización de este diagnóstico en la vida fetal para la temprana intervención al momento del nacimiento. Presentamos el caso de un paciente, en quien se integró el diagnóstico de esta pentalogía al encontrarse en la ecografía prenatal un defecto en pared abdominal gigante, y falta de integridad diafragmática, demostrándose que aunque la pentalogía de Cantrell es un síndrome potencialmente letal, sobre todo si se asocia a otras malformaciones, no contraindica la realización de cirugías paliativas o correctivas según sea el caso. Y los estudios prenatales, y posnatales oportunos, direccionan la elección del mejor tratamiento.

\section{Referencias}

1.- Willius FA: Unusually early description of socalled tetralogy of Fallot. Proc Mayo Clin 1948; 23: 316.

2.- Qun-Jun D, Zhan G, Ze-Wei Z: Correct definition of pentalogy of Cantrell. J Perinat Med 2009; 37: 426.

3.- Vásquez Jiménez J, Muehler EG, Daebritz $S$, et al: Cantrell's syndrome: a challenge to the surgeon. Ann Thorac Surg 1998; 65: 1178-85.

4.- Van Praagh R, Weinberg PM, Van Praagh S: Malposition of the Heart. En: Moss A, Emmanouilides GC (eds). Heart Disease in Infants, Children and Adolescents, Williams and Wilkins, Baltimore. 1977: 394.

5.- Hornberger LK, Colan SD, Lock JE, Wessel DL, Mayer $J E J r$ : Outcome of patients with ectopia Cordis and significant intracardiac defects. Circulation 1996; (suppl II) $94: 32-7$.

6.- Agrawal N, Sehgal R, Kumar R, Bhadoria P: Cantrell's Pentalogy. Anaesth Intensive Care 2003; 31: 120.

7.- Correa-Rivas SM, Matos IL, García LF: Pentalogy Of Cantrell: a case report with pathologic findings. Pediatr Develop Pathology 2004; 7: 649-52.

8.- Leca F, Thibert M, Khoury W, Fermont L, Laborde F, Dumez Y: Extrathoracic Heart (ectopia cordis). Report of two cases and review of the literature. Int J Cardiol 1989; 22: 221-8.

9.- Amato JJ, Zelen J, Talwalkar NG: Single-stage repair 
of thoracic ectopia Cordis. Ann Thorac Surg 1995; 59: 518-20.

10.- Medina $G$, Reyes $M$, Arteaga M: Ectopia cordis: autopsy findings in four cases. Pediatr Pathol 1991; 11: 85-95.

11.- Fernández IS, López A, Vila JJ, Lluna JM: Cantrell's pentalogy. Report of four cases And their management. Pediatr Surg Int 1997; 12: 428-31.

12.- Repondek Liberska M, Janiak K, Wloch A: Fetal Echocardiography in ectopia cordis. Pediatr Cardiol 2000; 21: 249-52.

13.- Zimmer EZ, Bronshein M: Early sonographic diagnosis Of fetal midline disruption syndromes. Prenat Diagn 1996; 16: 65-9.

14.- Haller JA, Cantrell RJ: Diagnosis and surgical correction Of combined congenital defects of supra-umbilical Abdominal wall, lower sternum and diaphragm. J Thorac Cardiovasc Surg 1966; 51: 286-91.

15.- Vipul KS, Usha K, Jyoti S, Poonam MK, Nitaa S: Challenges In the management of ectopia cordis. J Cardioth
Vasc Anesth 2001; 15: 618-23.

16.- Amato J, Zelen J, Talwalkar N: Single-stage Repair of thoracic ectopia cordis. Ann Thorac Surg 1995; 59: 51820.

17.- Shamberger RC, Welch KJ: Sternal defects Pediatr Surg Int 1990; 5: 156-164. 1990; 25: 726-30.

18.- Fernández MS, López A, Vila JJ, Lluna J: Cantrell's Pentalogy. Report of four cases and their management. Pediatr Radiol 1997; 12: 428-31.

19.- Gruberg L, Goldstein SA, Pfister AJ, et al: Cantrell's Syndrome: left ventricular diverticulum in an adult patient. Circulation 2000; 101 (1): 109-10.

20.- Grethel EJ, Hornberger LK, Farmer DL: Prenatal and Postnatal management of a patient with pentalogy of Cantrell and Left ventricular aneurysm. Fetal Diagn Ther 2007; 22 (4): 269-73.

21.- Halbertsma FJ, van Oort A, van der Staak F: Cardiac Diverticulum and omphalocele: Cantrell's pentalogy or syndrome. Cardiol Young 2002; 12: 71-4. 